\title{
Applications of the Galileo System in Civil Engineering
}

\author{
Prof. Leoš Mervart \\ Department of Advanced Geodesy \\ Faculty of Civil Engineering, CTU in Prague \\ E-mail: mervart@fsv.cvut.cz
}

Key words: Galileo System, Civil Engineering, Research plan MSM 6840770032

\section{Research plan description}

Subject and goal of the research plan

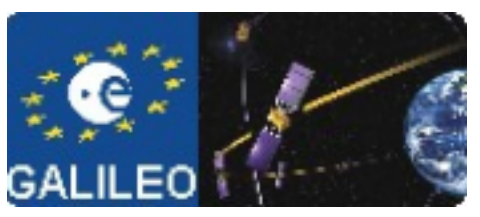

The development of the first satellite positioning systems also known as global positioning systems (GPS) - goes back to the 1960s. Their significance for technical practice started to increase together with gradual completion of the NAVSTAR (Navigation Satellite Timing And Ranging) GPS system during the 1980s. Since then, the number of applications has been continuously growing, and GPS have become indispensable in various areas of human activity. With the development of new GPS applications, however, certain limitations of the presently single fully operational system, NAVSTAR GPS, have started to be manifested, which are mainly caused by the fact that the system was originally designed for the needs of the US armed forces, and its inventors did not bear in mind the hundreds of its various civil applications.

For this reason, one of the priorities of the European Union member countries was to develop its own satellite positioning system, which (unlike the United States' system) would be primarily designed for a whole range of very diverse civil applications. During the complicated and long preparatory phase of the project, both its overall concept and the name of the positioning system itself went through changes. Negotiations between the European Union and the United States, aimed at ensuring so-called interoperability of the existing and the new positioning system, were also difficult. At the present time, it may be stated that there are no more political obstacles on the way to the construction of a European positioning system, and its detailed concept has been approved. In 2006, the project advanced from the so-called development phase to the implementation phase. The operating phase should be reached in 2008. The positioning system of the European Union is based on two projects:

\section{EGNOS (Euro Geostationary Navigation Overlay Service) Project.}

This is a joint project of the European Space Agency (ESA) and the European Commission, which will (as based on a plan of 2005) comprise three geostationary satellites.

\section{GALILEO Project.}

The GALILEO system (also representing a joint ESA and European Commission project) is a highly ambitious project, which, after its completion, should represent the latest technology for precise positioning. From July 1st 2003 to the end of 2005, the project was in the socalled development phase, while 2006 set off its so-called implementation phase, and from 
2008 on the system should be fully functional. The system involves a total of 30 satellites on three orbits (inclination to the equator - 56 degrees, distance from the Earth - $23616 \mathrm{~km}$ ). The agreement on "the support, deployment and exploitation of satellite positioning systems GALILEO and NAVSTAR GPS" signed between the USA and the European Union in 2004 (on June 26th) has cleared the way for user interoperability and radiofrequency compatibility of both systems.

The GALILEO system presently enjoys one of the top priorities in all EU member states. The Czech Government decree No. 218 of 23.2.2005 on organizing active participation of the Czech Republic in the Galileo programme declares the readiness of the Czech Republic to support its own business and research subjects.

The design of services and signals within the GALILEO system was subject to long discussions and numerous changes. At the present time, however, it is evident that one of the signals of the GALILEO systems will be in the L1 high-frequency range. This fact makes way for simple and cheap receivers capable of working with both systems - NAVSTAR GPS as well as GALILEO. At the same time, this paves the way for considerable precision and mainly reliability improvement in positioning while using a combination of measurements coming from both navigation systems. A direct consequence of this qualitative leap forward to be expected is tremendous development of new applications, including applications in civil engineering.

We presume that due to the above-mentioned facts, it is necessary to focus the efforts of research staff members of the Faculty of Civil Engineering, CTU, on the research and development of the Galileo system applications in the fields of geoinformatics, landscape and civil engineering. We are convinced that in order to launch a project of such research, we possess a top-quality research team composed of experts in the field of satellite positioning systems, informatics, geodesy, highway engineering, building constructions and other civil engineering branches. We are also convinced that the submitted project is appropriately targeted, well timed (in relation to the GALILEO system's construction), and that its implementation will bring considerable economic benefits.

The intended research plan consists of three mutually linked up parts:

1. Research and development of methods for the processing of signals from Galileo satellites, problems of combining measurements coming from the Galileo system with the existing global positioning system NAVSTAR GPS, specific features related to the Galileo system's applications in the Czech Republic and linkage to current observation grids of the Czech Republic.

2. Research and development of efficient processing methods of position-related information provided by means of the Galileo system, its visualization, development of database and information systems (GIS) based on the data obtained through the Galileo system.

3. Research and development of the Galileo system's application in individual civil engineering branches:

- monitoring of deformations of bridge structures 
- monitoring of displacements of building structures by combining measurements coming from the Galileo system with laser scanning methods

- building machinery control

- long-term monitoring of displacements of tram and railway tracks

- prevention of risks in transporting hazardous freight

- searching and development of new applications of satellite positioning systems in civil engineering

- searching and development of new applications of satellite positioning systems and methods of remote sensing of the Earth in water management.

One of the principal targets of the submitted research plan, therefore, is complex development of civil engineering applications of the Galileo system. The term "civil engineering applications" denotes various technological procedures which imply obtaining position-related information on building structures, building machinery control, optimization of logistic problems during construction processes etc. as their component part. These will, on the one hand, include applications, which are presently being solved by using other technologies (e.g. terrestrial measurements). In such cases, the objective will be to develop technological procedures based on the Galileo system, which are more cost-efficient, more precise or less risky for the staff executing the measurements. On the other hand, the qualitative leap in the precision and reliability of positioning, incorporated in the Galileo system, will pave the way for completely new applications and technologies, which otherwise could not be made available by using existing means. These applications cannot be predicted one by one, and thus enumerated within the research plan proposal. Nevertheless, we presume that it is in particular the search for them and their development that should become a significant part of the proposed research plan.

The submitted project, however, is not focused solely on the application level of the Galileo system. The project is submitted by staff members of the Faculty of Civil Engineering, CTU, representing, to a large extent, surveyors and cartographers (Geodesy and cartography being one of the branches studied at the Faculty). Therefore, another major objective of the project is the development of algorithms, methods and software applications for processing original measurements coming from the Galileo system, and integration of the results obtained by means of this system into information systems. Individual applications in civil and landscape engineering may be linked up only to the results of this development.

The project, therefore, represents a certain synthesis of research in the branch of satellite surveying and geoinformatics with specific applications in civil and landscape engineering.

Present level of knowledge and research activity in sphere which is subject of the research plan, from both international and national standpoints

The part dealing with the subject and goal of the research plan makes it clear that in order to achieve the project's objectives knowledge coming from more branches of science and 
technology is necessary. This section gives a brief outline of the present level of research activity and knowledge in the fields essential for solving the research plan:

\section{Satellite surveying and navigation}

Satellite surveying, global positioning systems theory and development of algorithms and software tools for the processing of satellite observations is a kind of "starting point" of the submitted project as satellite observations represent the initial data source on which all intended applications are based. Satellite surveying itself cannot exist without links to other specializations falling under the branch of so-called geodetic surveying. Their common task is to allow positioning of static or moving objects within an exactly defined coordinate and time system. As any positioning is based on measurements carried out in a specific physical environment, study of physical characteristics of the Earth and study of time-related changes in these characteristics are also component parts of geodetic surveying.

The problems of satellite navigation have been a subject of interest of the staff members of the Department of Advanced Geodesy of the Faculty of Civil Engineering, CTU, since the start of the 1990s. The Head of Department, Prof. Mervart, is a co-author of two significant software systems for processing NAVSTAR GPS observations - so-called Bernese GPS Software (in cooperation with the Astronomical Institute, University of Berne, Switzerland) and the RTNET (Real-Time Network) programme used by the Japanese Institute of Geography for monitoring the Japanese Geonet network (a network of approximately 1200 permanent GPS stations).

Prof. Mervart and Dr. Lukeš are authors or co-authors of numerous scientific publications devoted to global positioning systems.

Other research team members - Prof. Kostelecký, Dr. Vondrák, and Dr. Pešek are experts in the field of the national reference frame, geodetic astronomy and coordinate and time reference systems. The problems of reference frames are an important part of research as individual positioning systems can work in variously defined and implemented reference frames whose correct transformation and subsequent conversion of results into systems used in the Czech Republic is the necessary condition for the use of satellite positioning systems for precise applications in technical practice.

Research activity in the field of satellite surveying is indispensable without wide international cooperation. The above-mentioned research team members are engaged in international cooperation projects in the framework of bilateral agreements with our foreign partners (mainly the Astronomical Institute, University of Berne) and international scientific organizations mainly International GNSS Service and International Earth Rotation and Reference systems Service (IERS).

Among domestic research workplaces, our partner is mainly the Research Institute of Geodesy, Topography and Cartography.

\section{Informatics, geoinformatics, digital cartography and geographic information sys-} tems

The second pillar of the intended research plan is a group of sciences which (if we want to sum them up by a one-word term) may be referred to as "geoinformatics". This modern branch of science applies the knowledge of informatics - a science on processing and handling 
information - to fit the needs of geodesy, cartography and other scientific and technical disciplines dealing with measurements, mapping or (as is the case of civil engineering) also transformations of the earth's surface. In our concept, geoinformatics is a very wide term which serves, to a certain extent, as an umbrella for cartography, photogrammetry, remote sensing, mapping and land register. The significance of geoinformatics is also testified by the fact that "Geoinformatics" is the name and content of a newly accredited branch of study at the Faculty of Civil Engineering, CTU. Instruction in this branch will start in the 2006/2007 academic year.

The Department of Mapping and Cartography of the Faculty of Civil Engineering, CTU, is a top workplace in the fields falling under geoinformatics. The Department also comprises the Laboratory of Remote Sensing, whose research is focused on several areas. One of them is the monitoring of time-related changes in landscape, which may be determined from the data obtained through remote sensing - satellite data (optical and radar data) and aerial data (aerial photogrammetric images). The process of image evaluation necessarily requires precise localization of monitored changes. The Galileo system and the data provided by it will allow data acquisition with a substantially greater position precision and easier exploitation of the knowledge obtained in practice.

The Laboratory of Remote Sensing has been involved in the problems of differential interferometry for several years. This method allows collecting information on changes in the position of a territory on the earth's surface. A supporting tool for evaluating the results processed by differential interferometry is e.g. GIS where based on various sorts of input data a theoretical possibility of the existence of areas of subsidence is investigated. In this way, the values taken over from geological, mining and other background materials have been compared, which, however, cannot be used as absolutely reliable for confirming or denying subsidence. The Galileo system will allow continuous position monitoring in selected localities for several years. These measurements will be compared with the results of interferometric evaluation. The precondition, therefore, is to use the Galileo system for measurements on pre-selected localities. Such data will be regularly evaluated, and the information entered into GIS.

Another workplace of the Department of Mapping and Cartography of FCE, CTU, is the Laboratory of Photogrammetry. The laboratory activity in the last five years has been focused mainly on the use of terrestrial photogrammetry for documenting historical monuments where a number of significant achievements have been made while working on international projects as well. Higher forms of digital photogrammetric evaluation systems using the principles of virtual reality are represented in the laboratory at four stations. The pilot project of the laboratory in the long run is work on the PhotoPa system, which presently represents a relatively extensive photogrammetric and surveying database of minor historical monuments. Collecting this kind of data involves geoinformation elements, and the European GALILEO positioning system is presumed to be used for locating the objects.

\section{Engineering geodesy}

Engineering geodesy is an application of geodetic methods in industry and civil engineering. Among the principal tasks of engineering geodesy there is complete geodetic site management - from works carried out during the design phase of construction through site surveying to documentation of its as-built version and, in some cases, even long-term monitoring of its 
shifts and deformations.

Engineering geodesy is characterized by high demands for measurement precision and also by the fact that the measurements are carried out in very difficult conditions. The use of the latest devices is often the only way to fulfil the accuracy requirements observing, at the same time, the health and safety rules and reducing the risks of occupational accidents. In this respect, the use of satellite positioning systems in laying out major construction projects, the laser scanning method or the methods of automated building machinery control must be mentioned. The current positioning system NAVSTAR GPS has already been successfully used in some of the applications mentioned above. The use of the Galileo system, however, would result in reaching a greater precision of results replacing thus classic terrestrial methods in engineering geodetic applications setting high demands mainly for precise positioning. The impact of the new Galileo system would be still more significant in the cases where measurements are carried out in unfavourable conditions (e.g. limited visibility due to existing development etc.). With the number of satellites being more than twice bigger (a total of 54 satellites in simultaneous application of both the NAVSTAR GPS and the Galileo systems as compared to only 24 NAVSTAR GPS satellites) highly precise measurements under such difficult conditions would be feasible.

The problems of on-site geodetic measurements are studied by the Department of Special Geodesy of the Faculty of Civil Engineering, CTU, and also by the Department of Geodesy and Land Consolidation, FCE CTU. Doc. Blažek, the Head of the latter Department, is engaged in measurements of bridge deformations using optical methods. Ing. Stroner, PhD. from the Department of Special geodesy, FCE CTU, deals with the method of laser scanning of structures. Doc. Hampacher is an expert in mathematical processing of geodetic measurements using the adjustment calculus.

\section{Building mechanics, highway engineering, environmental engineering, water man- agement and water structures}

The Department of Building Mechanics of the Faculty of Civil Engineering, CTU, has been, among other things, involved in the long-term monitoring of static and dynamic behaviour of prominent building structures and detection of their excessive static deformations and dynamic deflections. The current accuracy of satellite observations using NAVSTAR GPS is, as a rule, lower that the precision required during the above-mentioned monitoring. It may, however, be expected that after higher accuracy has been achieved by introducing the Galileo system, terrestrial measurements may be, in some cases, replaced with satellite observations with considerable economies and enhanced health and safety. Evaluation of the results achieved would be greatly facilitated by the fact that repeated measurements could be replaced with measurements performed by permanent satellite receivers eliminating thus the effect of periodic phenomena (e.g. temperature fluctuations during the day or year) on the total determined behaviour of building structures.

The Department of Railway Structures, FCE CTU, among other things, deals with monitoring displacements of selected sections of tram and railway tracks - e.g. trial sections with new structural elements. These displacements are presently monitored by means of terrestrial methods. The use of satellite methods would be greatly beneficial both in terms of cost-effectiveness and health and safety. The condition is not only high precision of the measurements, but also a capability of reaching this high precision rate in conditions standard 
for railway and tram tracks - limited visibility of satellites in cuttings, impaired reception of signals due to vegetation etc.

The Department of Sanitary and Ecological Engineering, FCE CTU, is engaged in modelling distribution networks and rainfall-runoff processes in urbanized watersheds and evaluation of the ecological state of water courses and water/supply reservoirs, which requires precise information on the type and size of surfaces in the respective watershed, including their altitudinal surveys and boundaries and delimitation and precise location of structures, e.g. surface attributes of drinking water supply systems and sewage systems, which could be easily made available by using satellite methods.

The Department of Steel and Timber Structures, FCE CTU, participates in solving tasks aimed at monitoring steel and timber structures showing increased risks of excessive deformations and shifts. Such hazards are characteristic e.g. of historic spire timber roof structures. The majority of structures suffer from gradual material degradation of the bearing structure and in extreme climatic conditions (wind load) there are risks of their failures and irreparable damage. That is why measurements applying the proposed method and millimetre precision rate are of invaluable here, and they may very well identify structural failures. The use of satellite methods for these structures is beneficial as these structures are usually taller than the surrounding development. For this reason, any other measurement in such conditions sets high demands, while satellite receivers, on the contrary, provide considerable benefits as there is no danger of the receivers being shaded.

The use of the proposed method would also significantly enhance measurements on high masts, towers, chimneys and other similar structures. In such structures, second limit state (serviceability limit state) is often relevant. Here, dynamic wind effects are mostly in question, which must be monitored both in the actual wind direction and in the perpendicular direction, and cylindrical structures in particular may show resonance vibrations due to wind effects and be prone to fatigue damage. Wind effects may be, in a very complicated way, monitored in wind tunnels (scale models are used) where actual wind effects are only simulated. Measurements using satellite receivers would provide very valuable information on the basis of which actual wind effects could be analysed on real structures. The proposed project would significantly affect development in this area of structural design.

The Department of Geotechnics is ready to participate in solving the research plan by using knowledge referring to foundation condition of structures (rock mass condition in underlaying subsoil of buildings) and their foundation structures, including geotechnical causes of structural failures. Shape deformations of buildings may be also caused by rock mass dynamics, regardless of the fact if these are autonomous processes in the massif or part of interaction of the massif with buildings. Reliable results can be obtained only by complex assessment. The Department is well equipped by material and staff to monitor and evaluate the rock mass dynamics in interaction with buildings. Cooperation with other participating researchers is expected to result in obtaining valuable geodetic and geotechnical background materials for the evaluation of various types of localities; the research team of the Department of Geotechnics will also provide engineering geological and geotechnical background materials for other planned works. 


\section{Sub-goals of the research plan}

\section{System analysis and setting principal goals (SA)}

The process is a follow-up to the results achieved by the researchers prior to the project's launch. It runs at the start and, at the same time, during the whole time of the project duration to ensure that the partial tasks set are updated in relation to international development in the given problem area. A system solution to the project comprises a basic analysis of problems to solve, an application analysis and subsequent setting of demands for the functionality and properties of subsystems to ensure mutual linkage of individual processes.

\section{Research, development, testing and optimization of computation algorithms for processing information from Galileo satellites (GA)}

The process is one of principal tasks of the proposed research plan. It is a follow-up to previous researchers' works dealing with programme development for processing NAVSTAR GPS measurements in real time. In this part of the research plan we intend to:

- develop a programme for processing so-called phase measurements of the Galileo system

- assess the effect of corrections of the EGNOS system on the determined position of the receiver, design an optimum method of using the EGNOS system for intended technical applications

- investigate problems related to a combination of NAVSTAR GPS and Galileo measurements (different reference systems etc.) and prepare software tools for solving these problems

A new generation of satellite signal receivers of is presently being prepared. These new receivers differ from the existing ones in that some hardware elements are replaced with firmware elements. The receivers will be fitted with efficient processors and will become, to a certain extent, universal. By modifying or changing firmware, the receiver will be able to be used for measurements with different global positioning systems or their combinations (NAVSTAR GPS, Galileo and potentially others). The receivers will possess their own operating system allowing running user programmes directly in the receivers. This paves the way for developing new applications to optimize processing original raw data, solve communication with other devices within a given technology etc. Research and development of such applications, which are on the boundary line between firmware and user applications, will also form part of our work.

\section{Integration of national reference frame into common European reference frame (PZ)}

Research is targeted on the implementation of the European terrestrial reference frame ETRF89 on the territory of the Czech Republic with centimetre precision and its linkage to existing centimetre precision horizontal and vertical coordinate control systems (S-JTSK, S43/83, ČSNS). Only by observing this standard will it be possible to provide localization in all coordinate reference frames with the desired accuracy. As satellite movement is primarily described by means of laws referring to celestial mechanics in a celestial quasi-inertial celestial system, it will also be necessary to refine precision in transformation relations between this celestial and the rotating terrestrial reference system (precession, nutation, universal time, 
pole movement). Some parameters of the Earth orientation (precession, nutation, universal time), however, cannot be obtained only from satellite observations, and a combination with VLBI observations is necessary. Therefore, research will be also aimed at developing the technique of these combinations. To build the integrated terrestrial reference frame requires, apart from other prerequisites, refining information on the quasigeoid surface on the domain of the Czech Republic by processing heterogeneous data, verifying its precision based on the "GPS-levelling" method, developing an algorithm of ETRF89 - S-JTSK and ETRF - S42/83 transformation and supplying relevant software with regard to its incorporation into GIS software.

\section{Applications of modern methods of numerical mathematics (NM)}

Study of effective and numerically stable methods for solving mathematical problems that occur in processing observations from satellite positioning systems and in processing huge geoinformation files. The research plan proposal does not make it possible to precisely predict which mathematical problems are going to arise during the project solution. Generally we can expect that we will face problems stemming from solutions of large linear equation systems (when processing observations from satellite positioning systems the rank can be expected in the order of 100 thousand unknown). What is demanded is not only high numerical stability, but also high solution speed. Optimal algorithms can be achieved when a-priory knowledge of the linear equation system structure is taken into account (e.g. in the case of "sparse" equation systems or systems with some parameters strongly dependent etc.) When processing satellite data in real time various modifications of the Kalman filter are mostly used (the main reason is effectiveness of the solution). In general, we can say that such filtering can be numerically highly unstable. Optimization of filtering algorithms in terms of numerical stability is another goal of this project section.

\section{Monitoring of deformations of bridge structures (MK)}

This area is considered as one of the most promising applications of satellite observations in the building industry. The objective of the project is verification of the serviceability of the new European Galileo positioning system for the monitoring of static and dynamic behaviour of significant building structures and for the detection of their excessive static deformations and dynamic deflections.

In terms of serviceability, operating ability, long-term reliability and durability of large-span pre-stressed bridge structures, the highly topical issue of today is the problem of time-related permanent deformation growth. Social relevance of the problems affecting the operating ability of bridges is immense, and the resulting costs economically greatly exceed the costs due to static failures.

Experience shows greater values of real deflections as compared to predictions based on calculations and their long-term growth in time - actual long-term deflections are greater than those specified by standard calculations. The causes of this phenomenon are many, and they need to be objectively analysed.

One (though not the only) significant factor affecting deflection development is creep and differential shrinkage of concrete. Due to the fact that these are highly complex phenomena including interaction of a number of factors at different microstructure levels, which are affected by multiple variables, the mathematical expression of the development of these 
phenomena is necessarily rather complicated.

The research team of the Department of Concrete Bridge Structures and Bridges, FCE CTU, has achieved concrete results representing both original achievements in the theory of building structures, and support for design practice and monitoring of the deformation development of large bridges. The achievements include new mathematical models and computation methodology, generalization of results and practical recommendations serving as an efficient tool for reliable and economical design of structures, for reaching economies of construction materials, energy and resources, not only for construction, but also for maintenance, repairs and reconstruction.

Information on the actual deformation time pattern of bridge structures of pre-stressed concrete is essential for the calibration of theoretic predictions. It may be used both for the assessment of condition of monitored bridge structures, but also for the verification of the truthfulness of mathematical models of creep prediction.

Example: Deflection development is monitored on the bridge on the D8 motorway over the Ohře River near Doksany village. An increment of permanent deflection of about $3 \mathrm{~cm}$ was measured in five years at the midpoint of the longest span of this bridge, which is 130 meters long. Such big deformations lend themselves to speculations about their measuring using the methods of satellite geodesy, for which, as compared to standard methods, at least one level higher precision, but also continuous monitoring during the entire long-term measurement period could be expected.

Load due to temperature variations is a significant part of the total stress acting on large building structures. Information on deformations of a structure caused by temperature changes that would be obtained by using the Galileo system and supplemented by measuring the temperature variations, which caused the deformations, may serve for refining the knowledge used in background materials, design models and techniques for calculations and judgement of the effect of temperature variations on the reliability of investigated structures.

Example: Today, temperature variations of several bridges in the Czech Republic are systematically being observed with the purpose of verification of values specified in the assumed European standard EN 1991-1-5. The Department of Building Mechanics, FCE CTU, is observing temperature variations of a pre-stressed concrete bridge over the Sedlický Brook on the D1 motorway in the middle cross-section of its longest span that is $75 \mathrm{~m}$ long. The deflection of this bridge structure due to temperature variations reaches $\mathrm{ca} 1 \mathrm{~cm}$.

It is evident from the above-mentioned facts that the Galileo system could be used for:

- monitoring of the increment of permanent deflections of important pre-stressed concrete bridges,

- monitoring of the growing capacity to yield of important building structures caused by gradual degradation of their bearing structure,

- real-time monitoring of changes of basic natural frequencies of important building structures induced by gradual degradation of their bearing structure,

- detection of abnormal static deformations of monitored structures caused by extreme static live load (e.g. snow), 
- detection of excessive vibrations of monitored structures caused by extreme dynamic live load (e.g. swinging of a monitored structure by extreme wind effect, swinging of a footbridge by vandals) or by loss of aerodynamic stability.

The first phase of research on the project presumes comparison of the data obtained by the Galileo system with the data measured by classic techniques (research workplace: Department of Geodesy and Land Consolidation, FCE CTU). Transition from classic measurements to satellite observations with the advantages of economic effectiveness, improved health and safety and greater reliability of results should be possible after the verification of results.

Part of the task is also the development of methods for mathematical-statistical processing of obtained data and the development of software support for computations.

\section{Monitoring of failures and excessive deformations of significant historical buildings, monitoring of deviations and deformations of tall masts, towers and chimneys}

The aim of this part of the project is to verify the serviceability of the new European GALILEO positioning system for the monitoring of static and dynamics behaviour of significant (mainly historical) building structures and for the detection of their excessive deformation and dynamic deflections, which usually become a signal of a failure in the bearing structure.

The risks of failures or damage are of principal importance e.g. in historically valuable timber structures of spire roofs. In the majority of timber structures, progressive degradation of the material of the main structure occurs (effects of humidity, wood-destroying insects etc.), and during a severe climatic exposure (for spire roofs mainly under wind load) there is a risk of a failure. Therefore, application of satellite receivers with millimetre precision would be very beneficial to identify very well the defect of the structure and also give answers to questions if rehabilitation (strengthening) of the bearing structure is necessary. The use of satellite methods is very useful in the case of above mentioned structures because these structures usually exceed in height the surrounding terrain and buildings. Any other measurements is in this case are very complicated and expensive, while application of the satellite methods is very favourable, because there is no risk of overshadow of receivers, there is no need for a permanent operator, the measurement can be restricted to only specific periods of time in which the structure is exposed to extreme effects.

Satellite observations represent one of a few possibilities how to verify the behaviour of tall masts, towers and chimneys and other similar structures exposed to wind effects. For these structures, the limit state of serviceability (so-called second limit state) is usually crucial. Here, dynamic effects of wind are mostly the cause which must be monitored both in the direction of acting wind and in the perpendicular direction where mainly cylindrical-shaped structures could be vibrated by resonance and consequently endangered by fatigue damage. Wind effects are very difficult to monitor on scale models in wind tunnels where real wind impacts are simulated. Measurements with the help of Galileo receivers would provide us unique information on the basis of which we could analyze the real wind impacts on aerial structures. With respect to the proportions of these structures, their height-width ratio is very unfavourable for verification in wind tunnels, and monitoring of above mentioned variables on the real structures will entail significant advances in this branch. 
A further contribution would be a possibility to make measurements on such structures, which bear a considerable number of aerial systems and where there is a tendency to increase their dimensions or quantity. This problem is increasingly common because it is an accompanying phenomenon of the development of mobile communication networks. There is a limited number of tall buildings and structures in built-up areas and usually there is no chance to build new tall structures there, and this results in an increase in effective aerial areas on existing structures. There are some limits to this development and risks of failures under extreme climatic load. That is why, with the help of satellite receivers, the present state behaviour and also the behaviour after increasing the effective aerial area could be monitored on selected structures. In the case of a significant deformation growth, it will be possible to decide if the technology menaces the safety and stability of the structure and possibly how big additional horizontal load will be permissible in terms of maintaining the reliability of the whole system

\section{Application of the Galileo system in landscape and water management engi- neering (VI)}

The research workplaces (Department of Irrigation, Drainage and Landscape Engineering, FCE CTU, and Department of Sanitary and Ecological Engineering, FCE CTU) are going to develop expert and warning systems serving as decision-making support in real time. They will refer, in particular, to the following fields:

- water management - monitoring critical situations such as accidents, floods etc.

- landscape engineering - mapping and monitoring of valuable landscape elements, nature and landscape conservation

- transport and its environmental impact

Exact measurements with the help of the Galileo system will be used for the improvement of horizontal and vertical data on surface attributes and for exact positioning of remote sensing data. Possible applications are in areas such as:

- exact location of surface attributes of drinking water supply systems and sewage systems in $\mathrm{x}, \mathrm{y}, \mathrm{z}$ coordinates enabling a rapid access to control elements and manholes for operation purposes and rapid failure actions (digitising of the topographic base does not convey the coordinate of height, geodetic measurements are exigent and expensive, surface attributes are often hard to access, especially due to dense traffic)

- data for rainfall-runoff models of water quantity and quality (type and size of surfaces in the watershed and their slopes),

- data for the assessment of the ecological state of streams, rivers and water-supply reservoirs (their surveying, monitoring of changes in channels, riparian zones and watershed cover, water-level fluctuation)

- monitoring of accidents affecting water resources, progress of eutrophication of reservoirs

- monitoring of the descent of urban utility networks resulting from surface declination due to mining, etc.

Both research workplaces plan to provide theoretic outputs - feasibility studies, designs of applications and assessment of the system's technological demands and, in cooperation with 
other working groups, definition of technical requirements necessary for the system, as well as outputs in the form of demonstrations of individual applications.

\section{Application of the Galileo satellite system in highway engineering (SI)}

Exact location in space and time is becoming an important part of road transport and highway engineering. Several applications can be used e.g. in management and heavy vehicle control, easier overload checks, automatic highway toll systems etc. One of the most important challenges in terms of road safety is precise location of overload, oversize and dangerous freight during transport. The advantage of the Galileo satellite system can also be used for highway repairs and maintenance. This includes e.g. optimization of winter snow removal as well as routine monitoring, pavement management systems and highway maintenance, including measurements of their flexible and unchangeable variables.

The research team at the Department of Road Structures, FCE CTU, will proceed with the development of an expert system, which would, on a complex basis, include the above mentioned issues using the Galileo system as a principal source of spatial data. Special attention will be focused on ongoing programs in Canada and south-eastern Asia where GPS is already used. However, the system proposed by the FCE will employ the latest technologies of the new positioning systems, including communications between the user / vehicle and the central operating system after detecting a crisis situation and reacting to this situation.

\section{Applications of the Galileo system in railway engineering (ŽS)}

The research workplace (Department of Railway Structures, FCE CTU) will be engaged in the development of applications of the Galileo system for long-term monitoring of spatial displacements of selected sections of tram and railway tracks. In this case, the Galileo system will replace technologies based on terrestrial measurements using a technology based on satellite observations resulting in greater cost-effectiveness and health and safety. Monitoring of displacements of tracks is necessary in cases when the tracks are built with new structural elements, on high-speed tracks and tracks endangered e.g. by slope movements resulting from human activity (i.e. technogeneous movements e.g. due to undermining etc.) The objective of automated monitoring is to ensure greater safety of rail-borne vehicle operation.

\section{Combination of the laser scanning method and the Galileo system (LS)}

The research workplace (Department of Special Geodesy, FCE CTU) deals with modern methods of the monitoring and documentation of constructions by laser scanning systems. It is a highly effective and modern method, which allows mass collection of spatial data and its post processing and visualization. The most important device of the whole system - a laser scanner - produces coordinates in the local coordinate system and so the results of the measurement have to be transformed into the national reference coordinate system or into an alternative well defined suitable coordinate system. To perform the transformation accurately and effectively, it appears to be suitable to develop a technology, which combines the laser scanning measurement and observation of control points with use of the methods of the European space positioning system Galileo. The proposed task comprises:

- research of possibilities of mass data collection with the use of laser scanning systems for construction documentation purposes,

- research of possibilities of mass data collection with the use of laser scanning systems for 
the purposes of deformation analysis of constructions and other structures of interest,

- processing, visualization and presentation of data acquired by laser scanning systems,

- research of possibilities of interconnecting the results of terrestrial laser scanning and the results of the Galileo system's observations,

- comparison and linking of terrestrial geodetic technologies and modern satellite positioning methods with a view to the European Galileo system,

- research of possibilities of data acquisition for the purposes of facility management,

- usage of 3D models in city information systems (MIS).

\section{Application of the Galileo system in experimental geotechnics (EG)}

The state of rock or soil environment and its possible impact on the structure or on existing objects is evaluated during geotechnical surveys. The frequent task is to find out discontinuities, weakened zones, voids, cavities and other underground in-homogeneities. All this is provided by georadar surveys, best in combination with other methods (superficial refraction seismicity, micro-gravimetry).

The usage of georadars allows to obtain invaluable information about the geological structure near the construction route, e.g. about the depth and relief of underlaying rock, lithological changes, occurrence of disturbance zones etc. It enables the localization of utility networks and underground objects. Based on the results of georadar surveys it is possible to optimise the construction route, to classify earthwork, to determine the most suitable technology, to determine the range of blasting work, to evaluate the stability of the area or the possible impact of the construction on the surroundings. The georadar has its use both during construction and after its finishing (e.g. in the assessment of compaction quality). Its indisputable advantage is that it allows gaining high-density measured data. While the distance between survey drills is often several hundred meters, the distance, when using the georadar is only several decimetres.

The usage of the georadar in connection with the Galileo system enables gaining exactly localised outputs of this survey by linkage to the coordinate system. It will be possible to create a 3D automated continual database of underlaying rock of extensive areas by combining a non-destructive method of geotechnical surveying and the Galileo system.

\section{Evaluation of strain risks in structure-rock massif interaction (GT)}

Research in this area encompasses investigation of historical buildings (castles - Kunětická hora), landfills, spoil heaps (Palivový kombinát Ústí nad Labem - Rabenov), dumps, undermined localities and underground structures (Research underground laboratory, Gallery Josef, Mokrsko), slope deformations (Čertovka - Ústí nad Labem - Vaňov), traffic structures (highways), hydrotechnical structures (rockfill dams etc.), industrial structures and engineering structures (bridges) exposed to natural and anthropogeneous deformations of the rock massif. Following archive study, a choice of sensitive localities in terms of existing movements will be prepared (slope deformations, erosion, flood-related transport and accumulation, sensitive subsoil types, human activities). After shortlisting the number of structures whose investigation is feasible taking into account the time available and personal limits, the structures of interest will be instrumented and monitored. Results will be related to the measurements 
carried out on the rock massif, or in tunnels and drill holes. Finally, conclusions will be formulated evaluating potential use of analogical measurements and monitoring of structures.

\section{Web services design for geoinformatics (WS)}

The research is targeted at the design of a general object-oriented system for geoinformatics, with the objective of integrating various heterogeneous geoinformation services under a unified user interface. The interface for each service will be described in XML based on WSDL language. A registered user will be able to access results of submitted tasks, the geoinformation system itself will be written in Java language and based on servlets and the database handler JDBC. Privileged users will be allowed to register their own services in the system.

\section{Referencing of state large- and medium-scale map series by using the Galileo system (MA)}

The use of observations from the satellite positioning system must go hand in hand with entering the results in the existing topographic base. On the territory of the Czech Republic there is a number of state map series produced in a total of four coordinate systems and three cartographic projections with an unusually diverse range of sheet marking and sheet line systems. To meet the needs of digital cartography, international exchange of cartographic data and generally all modern technical applications, seamless maps (i.e. a map as a whole not a set of map sheets) must be introduced in a projection and a coordinate system chosen by the user. Problems arise e.g. due to the fact that base maps are produced by scanning and the contacts of neighbouring sheets are not aligned and, besides, they have irregular shrinkage. The research workplace (Department of Mapping and Cartography, FCE CTU) will work on the development of tools for the conversion of the existing topographic base into a uniform (electronic) format so that it may be used jointly with the Galileo satellite positioning system.

\section{Application of the Galileo system for enhanced effectiveness of real estate register management $(\mathrm{KN})$}

Technical applications of the positioning system in civil engineering rely on flawless functioning of the new Real Estate Register Information System (ISKN) and on rapid provision of current data for its graphic section (SGI). In this respect, the problems to be solved are creation of fast outputs in the form of thematic maps for crisis management at the time of natural disasters or similar events. Localization of selected elements within the territory by means of the GALILEO system can be conveniently combined with image data (colour orthophotos) to specify and monitor land use by means of the IACS system, based on the EU requirements and regulations for providing subsidies for agricultural production.

\section{Application of the Galileo system in photogrammetry (FG)}

The Laboratory of Photogrammetry deals with a number of tasks related to precise positioning. The laboratory activity has, in the long run, been focused on documentation and presentation of historical monuments with the aim of creating an extensive virtual database of listed monuments on the Internet. The first successful attempts in this area have been achieved http://lfgm.fsv.cvut.cz, the prototype of a database of minor historical monuments PhotoPa is in functional condition. This database, in particular, should be complemented by options of making animations and virtual reconstruction of monuments, and it should be made accessible to general public in the form of a simplified database for virtual viewing 
and tourism. Because of storing extensive amounts of data in the GIS environment which must be localized, a definition point or points must be specified for each object. Up to now, the technique used for this activity was measuring of position from a detailed map or hiking GPS. Another presumed system level will be visualization of objects based on their position on the map. Here, the Galileo system can be applied in connection with a digital map. A similar approach may be used for long-term investigation of geoglyphs and petroglyphs in Peru, which has been running for several years in cooperation with HTW Dresden and INC Peru. Here, the methods of precise GPS must be used for documentation purposes in extreme desert and mountainous conditions. Another research activity presumes creation of a system for multi-purpose navigation through cultural monuments. Here, the digital topographic base can be used complemented by additional useful information. In the last several years, laser scanning has become a new technology of documentation and mass collection of 3D points from the surroundings. At the same time, dynamic methods have appeared applying the GPS system complemented by an inertial positioning system. Inertial unit-fitted systems belong to hi-tech devices and are used in aerial applications. Terrestrial dynamic systems are still in the development stage. The research objective in this case will be creation of a dynamic system for surveying terrestrial line areas mounted on a car of rail-borne vehicle. The system's core will be a Galileo receiver and a laser measuring head. The presumed result of the measurement will be a 3D model of the nearest surroundings of the passed section with a multi-purpose use. 